\title{
The 13th Annual Kids Care Fair
}

\author{
Gail Silverman
}

American Red Cross of Greater Los Angeles

\begin{abstract}
The Kids Care Fair is an annual community outreach event of the American Red Cross of Greater Los Angeles. Details of the 13th Annual Kids Care Fair held in August 2002 are discussed.

(C) 2003 Californian Journal of Health Promotion. All rights reserved.

Keywords: Red Cross, child health, Kids Care Fair, Los Angeles
\end{abstract}

The American Red Cross of Greater Los Angeles 13th Annual Kids Care Fair was dedicated to improving the quality of health for children in Southern California (AMRCGL, 2002a,b). These fair sites (AMRGL, 2002c) were open to all children and their families living in Southern California and were absolutely free. The fairs were held on August 23, 24 or 25,2002 at 20 locations in Los Angeles, San Bernardino, and Orange Counties. The location of these fairs ranged from very large, suburban medical centers to small, community centers that were staffed by local medical clinics. Additionally, both medical and non-medical volunteers totaling 2,664 volunteers staffed the fairs. Total attendance for the threeday event was 16,321 with 5,715 children being screened.

Each site was a one-day event and offered a full range of free immunizations (AMRCGL, 2002d) required for all children in California, and a variety of health education activities and health screenings. All screenings and immunizations were available to children and youth through 18 years. These sites offered childhood immunizations including: MMR (Measles, Mumps, Rubella), DTaP (Diphtheria, Tetanus, Pertussis), IPV(Polio), Hepatitis-B, Hepatitis-A and $\mathrm{HiB}$ (Haemophilus Influenza Type B). At select sites, the varicella (chickenpox) vaccine was offered. In total, 2,202 children were immunized with a total of 4,021 immunizations given. Additionally, six basic screenings were conducted: Registration; Height \& Weight;
Vision; Blood Pressure; Dental; and Family Health Review. Family Health Review provides one of the most valuable components of the fair, where families were directed to free or low-cost resources for ongoing health care. Sites also host a Kids Safety Center, a Mom \& Dad Resource Center, and an Asthma Education booth. New this year is an Asthma Education Brochure published by the Kids Care Fair office to cover asthma health information basics.

In addition to the basic stations, optional stations available at selected sites included: TB testing, dermatology, fingerprinting, podiatry, scoliosis, and hearing, among others. Various health education and awareness booths featured bilingual information available on a variety of topics such as nutrition, eating disorders, childcare, HIV/AIDS and prenatal health care. Fun, healthy activities such as: crafts booths, face painting, clowns, games, and fire trucks were popular attractions at most fairs.

Beginning in August, Southern Californians could obtain information about Kids Care Fair in two languages (English and Spanish) by calling a toll-free hotline number. Additionally, Union 76 service stations, Circle K stores and Sav-On Drugs featured Kids Care Fair site lists and posters. Site information could also be found on the tray liners at neighborhood McDonalds restaurants as well as the website for the American Red Cross of Greater Los Angeles. The Kids Care Fair for 2002 was made possible by the cooperative sponsorship of the Sav-On Drugs, the American Red Cross, the KABC-TV 
Los Angeles and others, and the generous contributions of local Southern California County Health Departments.

Not to neglect the adult population, the American Red Cross of Greater Los Angeles holds a similar outreach screening program called Health Fair Expo each Spring. This program also provides needed health screenings and a low cost blood chemistry profile that includes risk of cardiac disease, diabetes and prostate specific antigen screening among others.

\section{References}

American Red Cross of Greater Los Angeles. (2002a). Home page. Retrieved February 7, 2003, from http://www.acrossla.org/

American Red Cross of Greater Los Angeles. (2002b). Kids care fair home page. Retrieved February 7 , 2003, from http://www.acrossla.org/EDUCATION/kcf.html

American Red Cross of Greater Los Angeles. (2002c). Kids care fair 2002 site list [Brochure]. Retrieved February 7, 2003, from http://www.acrossla.org/EDUCATION/kcf-sitelist.pdf

American Red Cross of Greater Los Angeles. (2002d). Kids care fair your child's immunization [Brochure]. Retrieved February 7, 2003, from http://www.acrossla.org/EDUCATION/kcf-immuz.pdf

American Red Cross of Greater Los Angeles. (2003). Health fair expo home page. Retrieved February 7, 2003, from http://www.acrossla.org/EDUCATION/hfe.html

\section{Acknowledgements}

Kids Care Fair Sponsors

\begin{tabular}{|l|l|}
\hline \multicolumn{1}{|c|}{ Organization } & \multicolumn{1}{c|}{ Web Address } \\
\hline KABC-TV Los Angeles & http://abclocal.go.com/kabc/ \\
\hline Sav-On & $\underline{\text { http://www.savon.com/ }}$ \\
\hline American Red Cross of Greater Los Angeles & $\underline{\text { http://www.acrossla.org/ }}$ \\
\hline
\end{tabular}

Kids Care Fair Additional Sponsors

\begin{tabular}{|c|c|}
\hline $\begin{array}{l}\text { Organization } \\
\end{array}$ & Web Address \\
\hline McDonald's Operators Association of California & \\
\hline Entertainment Industry Foundation & http://www.eifoundation.org/ \\
\hline Los Angeles Times & http://www.latimes.com/ \\
\hline Union 76 & http://www.76.com/ \\
\hline Circle K & $\begin{array}{l}\text { http://www.circlek.com/cgi- } \\
\text { bin/b2cpt01/scripts/home.jsp?Site=ck }\end{array}$ \\
\hline The California Endowment & http://www.calendow.org/ \\
\hline Flanigan Farms Natural Foods & \\
\hline Van Nuys Charities & \\
\hline Aventis Pasteur & http://www.aventispasteur.com/ \\
\hline California Pistachio Commission & http://www.pistachios.org/ \\
\hline Bristol-Myers Squibb & http://www.bms.com/landing/data/index.html \\
\hline
\end{tabular}




\section{In Cooperation With:}

\begin{tabular}{|l|l|}
\hline \multicolumn{1}{|c|}{ Organization } & \multicolumn{1}{c|}{ Web Address } \\
\hline $\begin{array}{l}\text { Immunization Branch } \\
\text { California Department of Health Services }\end{array}$ & http://www.dhs.cahwnet.gov/ps/dcdc/izgroup/ \\
\hline Los Angeles County Immunization Program & http://lapublichealth.org/ip/index.htm \\
\hline Orange County Immunization Program & http://www.ochealthinfo.com/mcah/immuniz.htm \\
\hline Riverside County Immunization Program & $\underline{\text { http://www.rivcoph.org/disease/imms.htm }}$ \\
\hline San Bernardino County Immunization Program & $\begin{array}{l}\text { http://www.co.san- } \\
\text { bernardino.ca.us/pubhlth/phdata/ProgramDetail.asp? } \\
\text { ProgramName=Immunizations }\end{array}$ \\
\hline Ventura County Immunization Program & $\underline{\text { http://www.vchca.org/ph/nursing/iz/index.htm }}$ \\
\hline
\end{tabular}

$\underline{\text { Author Information }}$

Gail Silverman, R.N., Director Community Outreach Services American Red Cross of Greater Los Angeles 